\title{
On next-to-eikonal exponentiation
}

\author{
E. Laenen $^{\mathrm{a}}$, L. Magnea ${ }^{\mathrm{b}}$, G. Stavenga ${ }^{\mathrm{c}}$ and C.D. White ${ }^{\mathrm{d}}$ \\ aNikhef, Science Park 105, 1098 XG Amsterdam; \\ ITF, Utrecht University, Leuvenlaan 4, 3584 CE Utrecht; \\ ITFA, University of Amsterdam, Science Park 904, 1090 GL Amsterdam, The Netherlands \\ ${ }^{\mathrm{b}}$ CERN, PH Department, TH Group, 1211 Geneva 23, Switzerland; \\ Dipartimento di Fisica Teorica, Università di Torino, \\ INFN, Sezione di Torino, Via P. Giuria 1, I-10125 Torino, Italy \\ ${ }^{c}$ Fermi National Accelerator Laboratory, MS106, P.O. Box 500, IL 60510, U.S.A. \\ ${ }^{\mathrm{d}}$ Institute for Particle Physics Phenomenology, Department of Physics, \\ Durham University, Durham DH1 3LE, United Kingdom
}

The eikonal approximation is at the heart of many theoretical and phenomenological studies involving multiple soft gauge boson emissions in high energy physics. We describe our efforts towards the extension of the eikonal approximation for scattering amplitudes to the first subleading power in the soft momentum.

\section{Introduction}

It is well-known that soft gauge boson emissions often give rise to large corrections to hard scattering cross-sections. Generically, if $1-x$ is a dimensionless variable related to the energy carried by undetected soft gauge bosons in a given process, the differential cross-section receives perturbative corrections of the form

$$
\begin{aligned}
\frac{d \sigma}{d x}=\sum_{m, n} \alpha^{n}\left[c_{n m}\right. & \left(\frac{\log ^{m}(1-x)}{1-x}\right) \\
& \left.+d_{n m} \log ^{m}(1-x)+\ldots\right],
\end{aligned}
$$

where $\alpha$ is the coupling constant and generically $m \leq 2 n-1$. The first term contains contributions that are not integrable as $x \rightarrow 1$, displaying an IR divergence that must be cancelled by virtual corrections, and the ellipsis denotes terms that are suppressed by powers of $1-x$. When $x \rightarrow 1$, the convergence of the perturbative expansion breaks down and resummation becomes necessary: one would like to know the coefficients $\left\{c_{n m}\right\}$ and $\left\{d_{n m}\right\}$ for all values of $n$. Much is known about the $c_{n m}$ coefficients, which are related to the eikonal approximation for soft radiation. This amounts to taking in each diagram the leading power term when all soft gauge boson momenta $k_{i} \rightarrow 0$. A full understanding of the $d_{n m}$ coefficients requires the use of the nextto-eikonal (NE) approximation, in which $k_{i} \rightarrow 0$ for all but one gluon, whose momentum is kept to first subleading order in the scattering amplitude.

A crucial result for resummations based on the eikonal approximation is the fact that amplitudes for soft gauge bosons form an exponential ("exponentiate"). For abelian gauge theory this has been understood since the early 1960s [1. For non-abelian theories, remarkably, similar results hold $2,3,4,5]$.

We have revisited this exponentiation with an eye to extending the eikonal approximation, and the results that are built upon it, to next-toleading power in the soft energy/momentum. We begin by describing a simple ansatz which generalizes threshold resummation, incorporating some recent insights into the structure of NE terms in the QCD splitting functions, as well as some wellunderstood phase space effects. While this ansatz 
is successful in reproducing the bulk of NE terms for inclusive cross sections, it does not give the full answer. We then go on to describe recent more systematic attempts to organize all NE terms, using either a path integral or a diagrammatic approach.

\section{Extended threshold resummation}

The authors of the three-loop calculation of Altarelli-Parisi splitting functions [6] found a remarkable relation between eikonal and next-toeikonal contributions: taking Mellin moments of splitting functions, they noted that the coefficients of terms proportional to $\ln N / N$ are determined by the coefficients of eikonal logarithms, $\ln N$. Subsequently, Dokshitzer, Marchesini and Salam (DMS) 7, proposed a modified evolution equation for parton distributions that connects eikonal and sub-eikonal terms in the splitting function in a nontrivial way, providing a justification for the results of [6]. In [8], we used these results to extend the threshold resummation formulae of $9|10| 11$, including the modified DMS evolution equation, and taking into account threshold kinematics at NE level. For the Mellin moments of the Drell-Yan partonic cross section, $\widehat{\omega}(N)$, we proposed the resummed expression

$$
\begin{aligned}
& \ln [\widehat{\omega}(N)]=\mathcal{F}_{\mathrm{DY}}\left(\alpha_{s}\left(Q^{2}\right)\right)+ \\
& \int_{0}^{1} d z z^{N-1}\left\{\frac{1}{1-z} D\left[\alpha_{s}\left(\frac{(1-z)^{2} Q^{2}}{z}\right)\right]\right. \\
& \left.\quad+2 \int_{Q^{2}}^{(1-z)^{2} Q^{2} / z} \frac{d q^{2}}{q^{2}} P_{s}\left[z, \alpha_{s}\left(q^{2}\right)\right]\right\}_{+},
\end{aligned}
$$

where the $n^{\text {th }}$ order term in the DMS-improved space-like evolution kernel is given by

$$
P_{s}^{(n)}(z)=\frac{z}{1-z} A^{(n)}+C^{(n)} \ln (1-z)+\ldots,
$$

The function $\mathcal{F}_{\text {DY }}$ controls $N$-independent terms according to [11, and the function $D$ controls contributions from wide-angle soft radiation. The $1 / z$ factors in the argument of the coupling in the function $D\left(\alpha_{s}\right)$, as well as the upper limit of the $q^{2}$ integral, reflect a more accurate accounting of threshold kinematics, and also lead to $1 / N$ effects. For DIS we proposed a similar form.

To assess the quality of our proposal, we compare the expansion of Eq. (2) in powers of $\alpha_{s}\left(Q^{2}\right)$, up to two loops, with the exact results of [12, in terms of the coefficients $a_{n m}$ and $b_{n m}$ in the expression

$$
\begin{aligned}
\widehat{\omega}(N)= & \sum_{i=0}^{\infty}\left(\frac{\alpha_{s}}{\pi}\right)^{n}\left[\sum_{m=0}^{2 n} a_{n m} \ln ^{m} \bar{N}\right. \\
& \left.+\sum_{m=0}^{2 n-1} b_{n m} \frac{\ln ^{m} \bar{N}}{N}\right]+\mathcal{O}\left(\frac{\ln ^{p} N}{N^{2}}\right)
\end{aligned}
$$

where $\bar{N}=N \exp \left(\gamma_{E}\right)$. As expected, all $a$ coefficients are reproduced. At the $1 / N$ level we find the results shown in table 1. We see that the leading $1 / N$ terms $\left(b_{23}\right)$ are reproduced for each color structure, while an excellent approximation for the next-to-leading ones $\left(b_{22}\right)$ is reached, and even $b_{21}$ is well reproduced. A similar conclusion holds for the DIS case, where we could compare with 2-loop [13] and even 3-loop results [14.

Full agreement at NE accuracy is, however, not reached here, nor in other approaches 15/16]17, $18|1920| 21$. To this end, a deeper understanding of exponentiation at NE accuracy is called for.

\section{Path integral approach}

It is possible to cast the exponentiation all possible subdiagrams involving soft gauge boson exchanges between external charged energetic lines in terms of the textbook exponentiation of connected Feynman diagrams 22. This technique in fact encompasses both the eikonal and nextto-eikonal approximations.

To show how this works, consider the pathintegral representation of the free scalar Feynman propagator

$\Delta_{F}=[\mathrm{i}(S-\mathrm{i} \varepsilon)]^{-1}, \quad S=\left(-\square_{x}+m^{2}\right)$,

which in momentum space reads

$$
\begin{aligned}
\tilde{\Delta}_{F}\left(p_{f}^{2}\right) & =\frac{1}{2} \int_{0}^{\infty} d T \frac{\left\langle p_{f}|U(T)| x_{i}\right\rangle}{\left\langle p_{f} \mid x_{i}\right\rangle} \\
& =-\frac{\mathrm{i}}{p_{f}^{2}+m^{2}-\mathrm{i} \varepsilon} .
\end{aligned}
$$


Table 1

Comparison of exact and resummed 2-loop coefficients for the Drell-Yan cross section. For each color structure, the left column contains the exact results, the right column contains the prediction derived from resummation according to Eq. (2).

\begin{tabular}{|c|c|c|c|c|c|c|}
\hline & \multicolumn{2}{|c|}{$C_{F}^{2}$} & \multicolumn{2}{|c|}{$C_{A} C_{F}$} & \multicolumn{2}{|c|}{$n_{f} C_{F}$} \\
\hline \hline$b_{23}$ & 4 & 4 & 0 & 0 & 0 & 0 \\
$b_{22}$ & $\frac{7}{2}$ & 4 & $\frac{11}{6}$ & $\frac{11}{6}$ & $-\frac{1}{3}$ & $-\frac{1}{3}$ \\
$b_{21}$ & $8 \zeta_{2}-\frac{43}{4}$ & $8 \zeta_{2}-11$ & $-\zeta_{2}+\frac{239}{36}$ & $-\zeta_{2}+\frac{133}{18}$ & $-\frac{11}{9}$ & $-\frac{11}{9}$ \\
$b_{20}$ & $-\frac{1}{2} \zeta_{2}-\frac{3}{4}$ & $4 \zeta_{2}$ & $-\frac{7}{4} \zeta_{3}+\frac{275}{216}$ & $\frac{7}{4} \zeta_{3}+\frac{11}{3} \zeta_{2}-\frac{101}{54}$ & $-\frac{19}{27}$ & $-\frac{2}{3} \zeta_{2}+\frac{7}{27}$ \\
\hline
\end{tabular}

In Eq. (6) we may introduce a path-integral representation for the matrix element

$$
\begin{aligned}
\left\langle p_{f}|U(T)| x_{i}\right\rangle & =\mathrm{e}^{-\mathrm{i} p_{f} x_{i}-\mathrm{i} \frac{1}{2}\left(p_{f}^{2}+m^{2}\right) T} \\
\times & \int_{x(0)=0}^{p(T)=0} \mathcal{D} p \mathcal{D} x \mathrm{e}^{\mathrm{i} \int_{0}^{T} d t\left(p \dot{x}-\frac{1}{2} p^{2}\right)} .
\end{aligned}
$$

The path integral is over all paths $x(t)$ with associated momentum $p(t)$, starting at fixed position $x_{i}$ and ending with final momentum $p_{f}$. It is not hard to generalize this by including an abelian gauge field. One gets

$$
\begin{gathered}
\left\langle p_{f}|U(T)| x_{i}\right\rangle=\int_{x(0)=x_{i}}^{p(T)=p_{f}} \mathcal{D} p \mathcal{D} x \exp [-\mathrm{i} p(T) x(T) \\
+\mathrm{i} \int_{0}^{T} d t\left(p \dot{x}-\frac{1}{2}\left(p^{2}+m^{2}\right)+p \cdot A\right. \\
\left.\left.+\frac{\mathrm{i}}{2} \partial \cdot A-\frac{1}{2} A^{2}\right)\right] \cdot
\end{gathered}
$$

One may now express the $n$-point Green function correlating $n$ hard particles by assuming a factorized form (which is known to be exact in the eikonal approximation), where hard fields are implicitly integrated out, while the path integral over soft fields yields eikonal and NE Feynman rules for soft emissions. We write

$$
\begin{aligned}
& G\left(p_{1}, \ldots, p_{n}\right)=\int \mathcal{D} A_{s}^{\mu} H\left(x_{1}, \ldots, x_{n}\right) \\
& \times\left\langle p_{1}\left|(S-\mathrm{i} \varepsilon)^{-1}\right| x_{1}\right\rangle \ldots\left\langle p_{n}\left|(S-\mathrm{i} \varepsilon)^{-1}\right| x_{n}\right\rangle,
\end{aligned}
$$

where $H$ collects all hard interactions, and there is an implicit integration over the coordinates $x_{i}$.
Notice that the one-particle path integrals for each external line are functionals of the soft fields which are then integrated explicitly in Eq. (9). To extract a scattering amplitude, we must truncate the external lines. Each external line then carries a factor of the form

$$
\left(p_{f}^{2}+m^{2}\right)\left\langle p_{f}\left|(S-\mathrm{i} \varepsilon)^{-1}\right| x_{i}\right\rangle \equiv \mathrm{e}^{-\mathrm{i} p_{f} x_{i}} f(\infty),
$$

where, after carrying out the $\mathcal{D} p$ integration,

$$
\begin{aligned}
& f(\infty)=\int_{x(0)=0} \mathcal{D} x \exp \left[\mathrm { i } \int _ { 0 } ^ { \infty } d t \left(\frac{1}{2} \dot{x}^{2}\right.\right. \\
& +\left(p_{f}+\dot{x}\right) \cdot A\left(x_{i}+p_{f} t+x(t)\right) \\
& \left.\left.+\frac{\mathrm{i}}{2} \partial \cdot A\left(x_{i}+p_{f} t+x\right)\right)\right] .
\end{aligned}
$$

From the point of view of the path integral over $A_{s}$ in Eq. (9) this is a collection of 1-point vertices for $A_{s}, i$. e. source terms. The scattering amplitude now reads

$$
\begin{aligned}
& S\left(p_{1}, \ldots, p_{n}\right)=\int \mathcal{D} A_{s}^{\mu} H\left(x_{1}, \ldots, x_{n}\right) \\
& \quad \times \mathrm{e}^{-\mathrm{i} p_{1} x_{1}} f_{1}(\infty) \ldots \mathrm{e}^{-\mathrm{i} p_{n} x_{n}} f_{n}(\infty) \mathrm{e}^{\mathrm{i} S\left[A_{s}\right]} .
\end{aligned}
$$

Each external line factor contains $A_{s}$ sources distributed along paths which are summed over in the one-particle path integrals. The $A_{s}$ path integral then connects these sources with propagators in all possible ways. The resulting gauge boson subdiagrams are either connected (as in Fig. 1) or disconnected (as in Fig. 2). At this point we can use the textbook result that, after performing the $A_{s}$ path integral, the scattering amplitude 

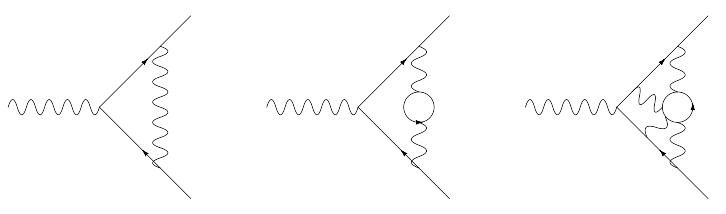

Figure 1. Examples of connected subdiagrams $G_{c}$ for soft emissions between hard outgoing particles in abelian perturbation theory.

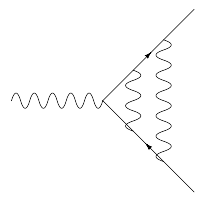

Figure 2. Example of a disconnected subdiagram between two outgoing external lines, to be compared with the connected subdiagrams of Fig. 1.

is the exponent of connected graphs. This gives a simple and direct proof of exponentiation for eikonal diagrams, and for a class of NE contributions: those that obey the factorization assumed in Eq. (91).

The combinatorics underlying this statement assume commuting sources. When considering a non-abelian gauge field this no longer holds, as each source carries a non-abelian charge. In order to use the same approach nevertheless, we employ a method that effectively shortcuts much of the combinatorial analysis for exponentiation: the so-called replica trick, which is used at times in statistical physics. Let us briefly sketch how the method works.

The Green functions of a given quantum field theory are described by the generating functional

$Z[J]=\int \mathcal{D} \phi \mathrm{e}^{\mathrm{i} S[\phi]+\mathrm{i} \int J \phi}$,

where $J$ is a source for the field $\phi$, and $S$ is the classical action. Now consider defining $N$ replicas of the theory, involving fields $\phi_{i}(i \in\{1, \ldots, N\})$. The generating functional becomes

$$
\begin{aligned}
Z_{N}[J]= & \int \mathcal{D} \phi_{1} \ldots \mathcal{D} \phi_{N} \\
& \times \mathrm{e}^{\mathrm{i} S\left[\phi_{1}\right]+\mathrm{i} \int J \phi_{1}} \ldots \mathrm{e}^{\mathrm{i} S\left[\phi_{N}\right]+\mathrm{i} \int J \phi_{N}},
\end{aligned}
$$

which clearly satisfies

$Z_{N}[J]=(Z[J])^{N}$.

The Feynman rules for each field are identical, and there are no interactions between the different replicas of the fields: thus, there can be no more than one field in each connected Feynman diagram, and connected diagrams come in $N$ copies. By the same reasoning, disconnected diagrams containing $k \geq 2$ connected components come in $N^{k}$ copies. It follows that the sum of all connected diagrams is proportional to the number of replicas, $\sum G_{c} \propto N$. Furthermore, no disconnected diagrams contribute terms proportional to $N$. From Eq. (15) one has

$Z_{N}[J]=1+N \log (Z[J])+\mathcal{O}\left(N^{2}\right)$,

which leads us to conclude that

$\sum G_{c}=\log (Z[J])$.

Finally, we may write this as

$Z[J]=\exp \left[\sum G_{c}\right]$,

and set $N=1$. For non-abelian gauge theory we note that although the fields are replicated, the gauge group is not, so that all replicas live in the same Lie algebra.

Let us consider the case of two external colored particles, corresponding to color-singlet process such as Drell-Yan. The scattering amplitude reads

$$
\begin{aligned}
S\left(p_{1}, p_{2}\right)=H\left(p_{1}, p_{2}\right) \int \mathcal{D} A_{s}^{\mu} & \\
& \times f_{1}^{i j_{1}}(\infty) f_{2}^{i j_{2}}(\infty) \mathrm{e}^{\mathrm{i} S\left[A_{s}\right]},
\end{aligned}
$$

where the external lines factors are path-ordered exponentials similar to Eq. (11). We can now use 
the combinatorial power of the path integral by replicating the gluon field, and defining a replicaordering operator $\mathcal{R}$ such that

$$
\begin{array}{r}
\prod_{i=1}^{N} \mathcal{P} \exp \left[\int d x \cdot A_{i}(x)\right] \\
=\mathcal{R} \mathcal{P} \exp \left[\sum_{i=1}^{N} \int d x \cdot A_{i}(x)\right],
\end{array}
$$

where

$\mathcal{R}\left[A_{i}(x) A_{j}(y)\right]=\left\{\begin{array}{ll}A_{i}(x) A_{j}(y), & i \leq j \\ A_{j}(y) A_{i}(x), & i>j\end{array}\right.$.

The classical path $x(t)$ in Eq. (11) is a straight line, leading to the standard eikonal approximation. By combining the two half-infinite paths into one path, we can categorize NE corrections using 1-dimensional field theory on the path. Two-point correlators correspond to path fluctuations, and lead precisely to NE Feynman rules for the soft gauge field in 4-dimensional Minkowski space.

The replica trick allows us to decide which diagrams connecting the sources on the path occur in the exponent, and, moreover, what their color factors are. In the eikonal approximation we recover precisely Gatheral's webs [234, with a non-recursive recipe to determine their modified color factors. Moreover, we identify at NE accuracy which new diagrams occur in the exponent (NE webs), and what their color factors are. Note however that not all NE corrections exponentiate: emissions that connect the hard function $H$ to the external lines, violating the factorization assumed in Eq. (9), and which are associated with the Low-Burnett-Kroll theorem [23 24,25], do not exponentiate. They can, however, be organized into an iterative pattern, as shown in [22].

\section{Diagrammatic approach}

For a different perspective on these results, one can follow a purely diagrammatic approach. For abelian gauge theory, in the eikonal approximation, one can consider all diagrams with an arbitrary number of photon exchanges (possibly via closed fermion loops). Such diagrams will in general contain disconnected pieces. Upon expanding all propagators and vertices to leading power in the soft momenta, and summing over all permutations $\pi$ of the emitted photons, one can decorrelate all photon emissions from each other by using the eikonal identity

$$
\begin{aligned}
& \sum_{\pi} \frac{1}{p \cdot k_{\pi_{1}}} \frac{1}{p \cdot\left(k_{\pi_{1}}+k_{\pi_{2}}\right)} \ldots \\
& \quad \times \frac{1}{p \cdot\left(k_{\pi_{1}}+k_{\pi_{2}}+\ldots k_{\pi_{n}}\right)}=\prod_{i} \frac{1}{p \cdot k_{i}} .
\end{aligned}
$$

Simple combinatoric arguments then show that the full amplitude $\mathcal{A}$, dressing the radiationless amplitude $\mathcal{A}_{0}$ with multiple soft photon radiation, takes the form

$$
\mathcal{A}=\mathcal{A}_{0} \sum_{\left\{N_{i}\right\}} \prod_{i} \frac{1}{N_{i} !}\left[G_{c}^{(i)}\right]^{N_{i}}
$$

where $G_{c}^{(i)}$ is a connected photon subdiagram joining the external lines, and $N_{i}$ is the multiplicity of this subdiagram occurring in the full diagram. Eq. (23) clearly displays an exponentation of the form of Eq. (18).

For non-abelian gauge theories one must use a generalization of the eikonal identity

$$
\begin{aligned}
\sum_{\tilde{\pi}} \frac{1}{p \cdot} k_{\tilde{\pi}_{1}} \frac{1}{p \cdot\left(k_{\tilde{\pi}_{1}}+k_{\tilde{\pi}_{2}}\right)} \ldots \\
\times \frac{1}{p \cdot\left(k_{\tilde{\pi}_{1}}+\ldots+k_{\tilde{\pi}_{n}}\right)} \\
=\prod_{g}\left[\frac{1}{p \cdot k_{g_{1}}} \frac{1}{p \cdot\left(k_{g_{1}}+k_{g_{2}}\right)}\right. \\
\left.\times \ldots \frac{1}{p \cdot\left(k_{g_{1}}+\ldots+k_{g_{m}}\right)}\right],
\end{aligned}
$$

where the product is over "groups" $g$, defined as projections of webs onto each eikonal line. The permutations $\tilde{\pi}$ are restricted to keep the ordering of gluon attachments within each group fixed. This product actually gives the set of eikonal gluon amplitudes the structure of a shuffle algebra 26. Using this algebraic structure one can set up an inductive proof of exponentiation, both for eikonal and NE approximations [27], which confirms and complements the discussion of [22]. 
The NE approximation, for those terms that exponentiate according to our discussion, can be implemented in practice through a set of effective Feynman rules. As a test, we used these rules to compute the abelian terms of the double-real emission contribution to the Drell-Yan process at two loops, we combined the result with the corresponding phase space and compared with the exact result [12]. We find

$$
\begin{aligned}
& K_{\mathrm{NE}}^{(2)}(z)=\left(\frac{\alpha_{s} C_{F}}{4 \pi}\right)^{2}\left[\frac{1024 \mathcal{D}_{3}(z)}{3}\right. \\
& -\frac{1024 \log ^{3}(1-z)}{3}+640 \log ^{2}(1-z) \\
& +\frac{512 \mathcal{D}_{2}(z)-512 \log ^{2}(1-z)+640 \log (1-z)}{\epsilon} \\
& +\frac{512 \mathcal{D}_{1}(z)-512 \log (1-z)}{\epsilon^{2}} \\
& \left.+\frac{256 \mathcal{D}_{0}(z)-256}{\epsilon^{3}}\right] .
\end{aligned}
$$

where $\mathcal{D}_{k}(z)$ are plus distributions, $\mathcal{D}_{k}(z)=$ $\left[\ln ^{k}(1-z) /(1-z)\right]_{+}$. The result is in complete agreement with [12].

\section{Conclusions}

How to resum sub-leading logarithms at nextto-eikonal accuracy is an unsolved problem, relevant for phenomenological applications [1628. An approach based on path integrals and using the replica trick yields much insight into exponentiation of these contributions in terms of (nextto-)eikonal webs, and it is corroborated by a diagrammatic analysis. We hope in future to extend our study to the level of full cross sections, and to processes involving more hard colored partons.

\section{REFERENCES}

1. D. R. Yennie, S. C. Frautschi and H. Suura, Annals Phys. 13 (1961) 379.

2. G. F. Sterman, AIP Conf. Proc. 74 (1981) 22.

3. J. G. M. Gatheral, Phys. Lett. B 133 (1983) 90.

4. J. Frenkel and J. C. Taylor, Nucl. Phys. B 246 (1984) 231.

5. C. F. Berger, arXiv:hep-ph/0305076.
6. S. Moch, J. A. M. Vermaseren and A. Vogt, Nucl. Phys. B 688 (2004) 101 arXiv:hep-ph/0403192.

7. Yu. L. Dokshitzer, G. Marchesini and G. P. Salam, Phys. Lett. B 634 (2006) 504 arXiv:hep-ph/0511302.

8. E. Laenen, L. Magnea and G. Stavenga, Phys. Lett. B 669 (2008) 173 arXiv:0807.4412 [hep$\mathrm{ph}]$.

9. G. F. Sterman, Nucl. Phys. B 281 (1987) 310.

10. S. Catani and L. Trentadue, Nucl. Phys. B 327 (1989) 323.

11. T. O. Eynck, E. Laenen and L. Magnea, JHEP $0306 \quad$ (2003) 057 arXiv:hep-ph/0305179.

12. R. Hamberg, W. L. van Neerven and T. Matsuura, Nucl. Phys. B 359 (1991) 343 [Erratum-ibid. B 644 (2002) 403].

13. E. B. Zijlstra and W. L. van Neerven, Nucl. Phys. B 383 (1992) 525.

14. J. A. M. Vermaseren, A. Vogt and S. Moch, Nucl. Phys. B 724 (2005) 3 arXiv:hep-ph/0504242.

15. A. Vogt, S. Moch, G. Soar and J. A. M. Vermaseren, arXiv:1001.3554 [hep-ph].

16. G. Soar, S. Moch, J. A. M. Vermaseren and A. Vogt, arXiv:0912.0369 [hep-ph].

17. S. Moch and A. Vogt, JHEP 0911 (2009) 099 arXiv:0909.2124 [hep-ph]].

18. G. Grunberg, arXiv:0710.5693 [hep-ph].

19. G. Grunberg and V. Ravindran, JHEP 0910 (2009) 055 arXiv:0902.2702 [hep-ph]].

20. G. Grunberg, Phys. Lett. B 687 (2010) 405 arXiv:0911.4471.

21. G. Grunberg, arXiv:1005.5684

22. E. Laenen, G. Stavenga and C. D. White, JHEP 0903 (2009) 054 arXiv:0811.2067 [hep-ph]].

23. F. E. Low, Phys. Rev. 110 (1958) 974.

24. T. H. Burnett and N. M. Kroll, Phys. Rev. Lett. 20 (1968) 86.

25. V. Del Duca, Nucl. Phys. B 345 (1990) 369.

26. S. Weinzierl, arXiv:1005.1855 [hep-ph].

27. E. Laenen, L. Magnea, G. Stavenga, C. D. White, to appear.

28. M. 1. Kramer, E. Laenen and M. Spira, Nucl. Phys. B $511 \quad$ (1998) 523 arXiv:hep-ph/9611272. 